\title{
Arbeit über Inventar zur Erfassung der Lebensqualität bei Kindern und Jugendlichen ausgezeichnet
}

Der Lilly Quality of Life-Preis 2006 wurde am 7. Dezember 2006 im Rahmen eines Workshops an vier Wissenschaftler verliehen: Dr. Elfriede Greimel aus Graz und Professor Dr. Andreas du Bois aus Wiesbaden sowie Professor Dr. Fritz Mattejat und Professor Dr. Dr. Helmut Remschmidt aus Marburg. Der mit insgesamt 10 000,- Euro dotierte Preis zur Lebensqualitätsforschung wird von Lilly Deutschland gestiftet.

Das Pharmaunternehmen Lilly Deutschland engagiert sich bereits seit 1996 jährlich mit der Ausschreibung des Lilly Quality of Life-Preises in der Lebensqualitätsforschung. Mit dem Preis soll die Integration der Lebensqualität in die Therapieentscheidung gefördert werden, um so die Lebensqualität von Patienten kontinuierlich $\mathrm{zu}$ verbessern und neue Wege der Behandlung zu finden. Die Preisträger wurden von einer fünfköpfigen Jury, bestehend aus Professor Elmar Brähler, Leipzig, Professor Gerd Glaeske, Bremen, Professor Thomas Kohlmann, Greifswald, Professor
Franz Porzsolt, Ulm sowie Dr. Nick Schulze-Solce, Direktor Corporate Affairs Lilly Deutschland, ausgewählt. „Wir freuen uns, dass sich immer mehr junge Wissenschaftler aus ganz unterschiedlichen Bereichen für die Verbesserung der Lebensqualität von Patienten engagieren“, resümiert Schulze-Solce. „In den letzten zehn Jahren konnten wir beispielsweise Arbeiten aus den Fachgebieten Psychiatrie und Neurologie, Zahnmedizin, Intensivmedizin und Anästhesie sowie der Onkologie auszeichnen.“

Die Preisträger des Lilly Quality-of-LifePreises 2006

Dr. Elfriede Greimel und Professor Andreas du Bois wurden für ihre Arbeit „Quality of Life in Patients with Ovarian Cancer Treated with Cisplatin/Paclitaxel versus Carboplatin/Paclitaxel“ ausgezeichnet, die sie mit ihren Kollegen aus der Arbeitsgemeinschaft Gynäkologische Onkologie/Studiengruppe Ovarialkarzinom realisierten. In der prospektiv randomisierten Phase-III-Studie verglichen die Preisträger die bisherige Standardtherapie des Ovarialkarzinoms (Cisplatin/Paclitaxel) mit einer neuen Kombinationstherapie (Carboplatin/Paclitaxel). Ihre Ergebnisse trugen dazu bei, dass sich inzwischen der neue Ansatz nicht zuletzt wegen der besseren Lebensqualität weltweit als Standard in der first-line Therapie durchgesetzt hat. Professor Fritz Mattejat und Professor Helmut Remschmidt stellten mit dem „Inventar zur Erfassung der Lebensqualität bei Kindern und Jugendlichen (ILK)“ ein Screening-Instrument bereit, das die Lebensqualität bei psychisch kranken, aber auch bei gesunden Kindern und Jugendlichen misst. Der so genannte Lebensqualitätsscore wird dabei durch Einschätzungen aus Sicht der Kinder und Jugendlichen, der Eltern sowie der behandelnden Therapeuten gewonnen. Die Ergebnisse dieser Einschätzungen fließen direkt und in systematischer Weise in die Therapieentscheidungen mit ein. 